\title{
Adaptive Minimum-BER Linear Multiuser Detection for DS-CDMA Signals in Multipath Channels
}

\author{
Sheng Chen, Senior Member, IEEE, Ahmad K. Samingan, Bernard Mulgrew, Member, IEEE, and Lajos Hanzo
}

\begin{abstract}
The problem of constructing adaptive minimum bit error rate (MBER) linear multiuser detectors is considered for direct-sequence code division multiple access (DS-CDMA) signals transmitted through multipath channels. Based on the approach of kernel density estimation for approximating the bit error rate (BER) from training data, a least mean squares (LMS) style stochastic gradient adaptive algorithm is developed for training linear multiuser detectors. Computer simulation is used to study the convergence speed and steady-state BER misadjustment of this adaptive MBER linear multiuser detector, and the results show that it outperforms an existing LMS-style adaptive MBER algorithm first presented at Globecom'98 by Yeh et al.
\end{abstract}

Index Terms-Adaptive algorithms, linear multiuser detectors, minimum bit error rate, minimum mean square error, stochastic gradient algorithms.

\section{INTRODUCTION}

W ITHIN the class of linear multiuser detectors for DS-CDMA signals, the minimum mean square error (MMSE) detector [1]-[5] is the most popular one as it provides good performance and can readily be implemented using standard adaptive filter techniques such as the LMS and recursive least squares algorithms [3], [5]. However, it is well known that the MMSE solution is not always optimal in this application, and the BER of the MMSE linear multiuser detector can, in certain situations, be distinctly inferior to the optimal MBER solution [6]-[9]. Gradient optimization for obtaining the theoretical MBER linear multiuser detector is considered in $[6]^{1}$ for narrowband Gaussian CDMA channels [i.e., channels that do not introduce intersymbol interference (ISI)]. Gradient optimization to achieve the theoretical MBER solution is also addressed in [7], where constraints are added to ensure a global solution by gradient-based algorithms at the expense that the solution obtained may not be the true MBER solution. There are two stochastic gradient adaptive algorithms for realizing the MBER linear multiuser detector in the literature [8], [9].

The adaptive algorithm given in [8] uses a difference approximation to estimate the gradient of one-sample error prob-

Manuscript received April 27, 2000; revised February 21, 2001. The associate editor coordinating the review of this paper and approving it for publication was Prof. Dimitrios Hatzinakos.

S. Chen, A. K. Samingan, and L. Hanzo are with the Department of Electronics and Computer Science, the University of Southampton, Southampton, U.K. (e-mail: sqc@ecs.soton.ac.uk).

B. Mulgrew is with the Department of Electronics and Electrical Engineering, the University of Edinburgh, Edinburgh, U.K.

Publisher Item Identifier S 1053-587X(01)03884-3.

${ }^{1}$ The "adaptive" algorithm in [6] is, in fact, nonadaptive as it requires the received signal minus the noise component. This is the same to an off-line optimization with the perfect channel knowledge and all user bits. ability and moves the detector weights in the negative direction of the estimated stochastic gradient. The algorithm only adjusts the detector weight vector when the detector makes an error. ${ }^{2}$ The main drawback of this algorithm is therefore a very slow convergence, particularly when the error rate is very low. For the sake of distinguishing this stochastic adaptive MBER algorithm from others, it will be called the difference approximation adaptive MBER (DMBER) algorithm. The adaptive algorithm reported in [9], which is called the approximate MBER (AMBER) detector, is appealing due to its computational simplicity. It is a stochastic gradient algorithm that is identical to the signed-error LMS algorithm [10], except in the vicinity of the decision boundary, where it is modified to continue updating the weights when the signed-error LMS algorithm would not. The AMBER algorithm therefore can continuously update when the detector weight vector has reached the regions of very low error rate.

Adaptive MBER linear equalizers have been investigated for a longer time [11]-[16]. In particular, the adaptive MBER equalizer presented in [15] and [16] is a LMS-style stochastic gradient algorithm and has been shown to outperform the approximate MBER linear equalizer first reported in [17], which is the counterpart of the AMBER linear multiuser detector of [9]. In this study, we extend the adaptive MBER algorithm of [15] and [16] to multiuser detection for DS-CDMA channels and develop a new adaptive MBER linear multiuser detector. For the purpose of distinguishing it from the two above-mentioned adaptive algorithms, this new LMS-style stochastic gradient adaptive algorithm will be referred to as the least BER (LBER) algorithm. Our investigation involving simulation shows that this new LBER linear multiuser detector is superior in performance over the AMBER linear multiuser detector of [9].

The paper is organized as follows. Section II presents the DS-CDMA system model used and provides the necessary notations and definitions. Section III is devoted to formulating the MBER solution for the linear multiuser detector and developing a gradient search algorithm. In Section IV, the proposed adaptive MBER multiuser detector is derived. Kernel density estimation is employed to approximate the BER as a smooth function of training data, and this leads to the formulation of a LMS-style stochastic gradient adaptive algorithm called the LBER. Comparison with the two existing stochastic adaptive algorithms (the DMBER and the AMBER) is also given in this section. Section $\mathrm{V}$ gives some computer simulation results, and the paper concludes in Section VI.

\footnotetext{
${ }^{2}$ More precisely, when the difference of the detector decisions corresponding to the weights perturbed in opposite directions is nonzero.
} 


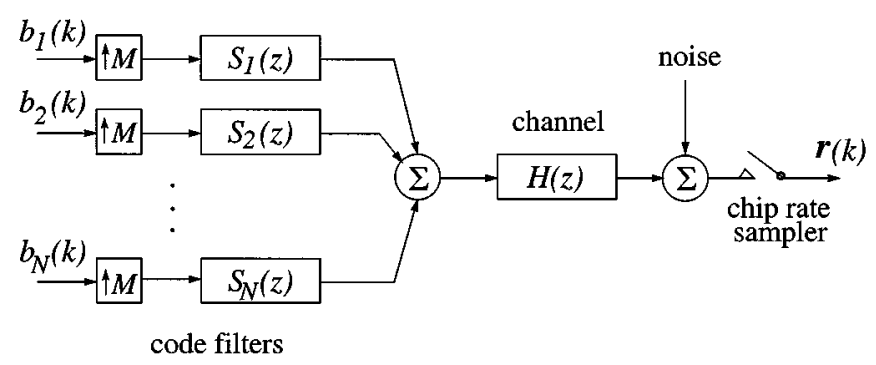

Fig. 1. Discrete-time model of synchronous CDMA downlink.

\section{SySTEM MODEL}

The discrete-time baseband model of the synchronous DS-CDMA downlink system with $N$ users and $M$ chips per symbol is depicted in Fig. 1, where $b_{i}(k) \in\{ \pm 1\}$ denotes the $k$ th symbol of user $i$, the unit-length signature sequence for user $i$ is

$$
\overline{\mathbf{s}}_{i}=\left[\bar{s}_{i, 1} \ldots \bar{s}_{i, M}\right]^{T}
$$

and the transfer function of the channel impulse response (CIR) is

$$
H(z)=\sum_{i=0}^{n_{h}-1} h_{i} z^{-i} .
$$

The baseband model for received signal sampled at chip rate is given by [4], [18]

$$
\begin{aligned}
\mathbf{r}(k)= & \mathbf{H}\left[\begin{array}{cccc}
\overline{\mathbf{S}} \mathbf{A} & \mathbf{0} & \cdots & \mathbf{0} \\
\mathbf{0} & \overline{\mathbf{S}} \mathbf{A} & \ddots & \vdots \\
\vdots & \ddots & \ddots & \mathbf{0} \\
\mathbf{0} & \cdots & \mathbf{0} & \overline{\mathbf{S}} \mathbf{A}
\end{array}\right] \\
\times & {\left[\begin{array}{c}
\mathbf{b}(k) \\
\mathbf{b}(k-1) \\
\vdots \\
\mathbf{b}(k-L+1)
\end{array}\right]+\mathbf{n}(k) } \\
= & \mathbf{P}\left[\begin{array}{c}
\mathbf{b}(k) \\
\mathbf{b}(k-1) \\
\vdots \\
\mathbf{b}(k-L+1)
\end{array}\right]+\mathbf{n}(k)=\overline{\mathbf{r}}(k)+\mathbf{n}(k)
\end{aligned}
$$

where $\overline{\mathrm{r}}(k)$ denotes the noise-free received signal; the white Gaussian noise vector

$$
\mathbf{n}(k)=\left[n_{1}(k) \ldots n_{M}(k)\right]^{T} \quad \text { with } E\left[\mathbf{n}(k) \mathbf{n}^{T}(k)\right]=\sigma_{n}^{2} \mathbf{I}
$$

the user symbol vector

$$
\mathrm{b}(k)=\left[b_{1}(k) \ldots b_{N}(k)\right]^{T}
$$

the user signature sequence matrix

$$
\overline{\mathbf{S}}=\left[\overline{\mathbf{s}}_{1} \ldots \overline{\mathbf{s}}_{N}\right]
$$

the diagonal user signal amplitude matrix

$$
\mathbf{A}=\operatorname{diag}\left\{A_{1} \ldots A_{N}\right\}
$$

that is, $A_{i}^{2}$ is the user $i$ signal power; the $M \times L M$ CIR matrix $\mathbf{H}$ has the form

$$
\mathbf{H}=\left[\begin{array}{ccccccc}
h_{0} & h_{1} & \cdots & h_{n_{h}-1} & & & \\
& h_{0} & h_{1} & \cdots & h_{n_{h}-1} & & \\
& & \ddots & \ddots & \cdots & \ddots & \\
& & & h_{0} & h_{1} & \cdots & h_{n_{h}-1}
\end{array}\right]
$$

and the $M \times L N$ system matrix

$$
\mathbf{P}=\mathbf{H}\left[\begin{array}{cccc}
\overline{\mathbf{S}} \mathbf{A} & \mathbf{0} & \cdots & \mathbf{0} \\
\mathbf{0} & \overline{\mathbf{S}} \mathbf{A} & \ddots & \vdots \\
\vdots & \ddots & \ddots & \mathbf{0} \\
\mathbf{0} & \cdots & \mathbf{0} & \overline{\mathbf{S}} \mathbf{A}
\end{array}\right]
$$

The channel ISI span $L$ depends on the length of the CIR $n_{h}$ related to the length of the chip sequence $M$. For $n_{h}=1, L=$ 1 ; for $1<n_{h} \leq M, L=2$; for $M<n_{h} \leq 2 M, L=3$; and so on. The model (3) adopted in this study is a wideband synchronous DS-CDMA channel, and it can be extended to the case of asynchronous DS-CDMA downlink systems.

We will study the linear multiuser detector of the form

$$
\hat{b}_{i}(k)=\operatorname{sgn}(y(k ; \mathbf{w})) \quad \text { with } y(k ; \mathbf{w})=\mathbf{w}^{T} \mathrm{r}(k)
$$

where

$$
\mathbf{w}=\left[w_{1} \ldots w_{M}\right]^{T}
$$

denotes the detector weight vector for user $i$. Let the $N_{b}=2^{L N}$ possible combinations or sequences of $\left[\mathbf{b}^{T}(k)\right.$ $\left.\mathbf{b}^{T}(k-1) \ldots \mathbf{b}^{T}(k-L+1)\right]^{T}$ be

$$
\mathbf{b}^{(j)}=\left[\begin{array}{c}
\mathbf{b}^{(j)}(k) \\
\mathbf{b}^{(j)}(k-1) \\
\vdots \\
\mathbf{b}^{(j)}(k-L+1)
\end{array}\right], \quad 1 \leq j \leq N_{b}
$$

and let $b_{i}^{(j)}$ be the $i$ th element of $\mathbf{b}^{(j)}(k)$. Define the $N_{b}$ noise-free received signal states

$$
\mathbf{r}_{j}=\mathbf{P b}^{(j)}, \quad 1 \leq j \leq N_{b}
$$

and the set of $N_{b}$ scalars

$$
y_{j}=\mathbf{w}^{T} \mathbf{r}_{j}, \quad 1 \leq j \leq N_{b} .
$$

\section{MBER Linear MULTIUSER Detector}

In this section, we derive the theoretical MBER solution for the linear multiuser detector and present a gradient search algorithm for finding this MBER solution. This will provide some insight into the development of our LMS-style stochastic gradient adaptive algorithm. The error probability of the linear detector (10) is

$$
P_{E}(\mathbf{w})=\operatorname{Prob}\left\{\operatorname{sgn}\left(b_{i}(k)\right) y(k ; \mathbf{w})<0\right\} .
$$


Following [15], [16], define the signed decision variable

$$
\begin{aligned}
y_{s}(k ; \mathbf{w}) & =\operatorname{sgn}\left(b_{i}(k)\right) y(k ; \mathbf{w}) \\
& =\operatorname{sgn}\left(b_{i}(k)\right) y^{\prime}(k ; \mathbf{w})+n^{\prime}(k)
\end{aligned}
$$

where

$$
y^{\prime}(k ; \mathbf{w})=\mathbf{w}^{T} \mathbf{P}\left[\begin{array}{c}
\mathrm{b}(k) \\
\mathbf{b}(k-1) \\
\vdots \\
\mathbf{b}(k-L+1)
\end{array}\right]
$$

and

$$
n^{\prime}(k)=\operatorname{sgn}\left(b_{i}(k)\right) \mathbf{w}^{T} \mathbf{n}(k) .
$$

Notice that $y^{\prime}(k ; \mathbf{w})$ can only take the values from the set defined in (14), and $n^{\prime}(k)$ is Gaussian with zero mean and variance $\sigma_{n}^{2} \mathbf{w}^{T} \mathbf{w}$. Under the assumption that $\mathbf{r}_{j}, 1 \leq j \leq N_{b}$, are equiprobable, the probability density function (pdf) of $y_{s}(k ; \mathbf{w})$ is

$$
\begin{aligned}
p_{y}\left(y_{s} ; \mathbf{w}\right)= & \frac{1}{N_{b} \sqrt{2 \pi} \sigma_{n} \sqrt{\mathbf{w}^{T} \mathbf{w}}} \\
& \times \sum_{j=1}^{N_{b}} \exp \left(-\frac{\left(y_{s}-\operatorname{sgn}\left(b_{i}^{(j)}\right) y_{j}\right)^{2}}{2 \sigma_{n}^{2} \mathbf{w}^{T} \mathbf{w}}\right) .
\end{aligned}
$$

Thus

$$
P_{E}(\mathbf{w})=\int_{-\infty}^{0} p_{y}\left(y_{s} ; \mathbf{w}\right) d y_{s}=\frac{1}{N_{b}} \sum_{j=1}^{N_{b}} Q\left(c_{j}(\mathbf{w})\right)
$$

where

$$
Q(y)=\frac{1}{\sqrt{2 \pi}} \int_{y}^{\infty} \exp \left(-\frac{x^{2}}{2}\right) d x
$$

and

$$
c_{j}(\mathbf{w})=\frac{\operatorname{sgn}\left(b_{i}^{(j)}\right) y_{j}}{\sigma_{n} \sqrt{\mathbf{w}^{T} \mathbf{w}}}=\frac{\operatorname{sgn}\left(b_{i}^{(j)}\right) \mathbf{w}^{T} \mathbf{r}_{j}}{\sigma_{n} \sqrt{\mathbf{w}^{T} \mathbf{w}}} .
$$

The gradient of $P_{E}(\mathbf{w})$ with respect to $\mathbf{w}$ is

$$
\begin{aligned}
\nabla P_{E}(\mathbf{w})= & \frac{1}{N_{b} \sqrt{2 \pi} \sigma_{n}}\left(\frac{\mathbf{w} \mathbf{w}^{T}-\mathbf{w}^{T} \mathbf{w} \mathbf{I}}{\left(\mathbf{w}^{T} \mathbf{w}\right)^{\frac{3}{2}}}\right) \\
& \times \sum_{j=1}^{N_{b}} \exp \left(-\frac{y_{j}^{2}}{2 \sigma_{n}^{2} \mathbf{w}^{T} \mathbf{w}}\right) \operatorname{sgn}\left(b_{i}^{(j)}\right) \mathbf{r}_{j} .
\end{aligned}
$$

The following steepest-descent gradient algorithm can be used to find the MBER solution:

$$
\mathbf{w}(l+1)=\mathbf{w}(l)-\mu \nabla P_{E}(\mathbf{w}(l))
$$

where $l$ indicates the iteration, and $\mu$ is an adaptive step size. Notice that the orientation of the weight vector $\mathrm{w}$ defines the decision boundary and, thus, the BER and not the size of $\mathrm{w}$. It is computationally advantageous to normalize $\mathbf{w}$ to a unit-length after every iteration:

$$
\mathbf{w}=\frac{\mathbf{w}}{\sqrt{\mathbf{w}^{T} \mathbf{w}}} .
$$

With this rescaling, the gradient can be simplified as

$$
\begin{aligned}
\nabla P_{E}(\mathbf{w})= & \frac{1}{N_{b} \sqrt{2 \pi} \sigma_{n}} \sum_{j=1}^{N_{b}} \exp \left(-\frac{y_{j}^{2}}{2 \sigma_{n}^{2}}\right) \\
& \times \operatorname{sgn}\left(b_{i}^{(j)}\right)\left(\mathbf{w} y_{j}-\mathbf{r}_{j}\right) .
\end{aligned}
$$

The steepest-descent gradient algorithm (24) may converge slowly, and a Gauss-Newton algorithm is computationally expensive. The conjugate gradient method [19] offers a better alternative. A simplified conjugate gradient algorithm is summarized.

Initialization: Choose step size $\mu>0$ and termination scalar $\beta>0$; given $\mathbf{w}(1)$ and $\mathbf{d}(1)=-\nabla P_{E}(\mathbf{w}(1))$; set $l=1$.

Loop: If $\left\|\nabla P_{E}(\mathbf{w}(l))\right\|=\sqrt{\left(\nabla P_{E}(\mathbf{w}(l))\right)^{T} \nabla P_{E}(\mathbf{w}(l))}<$ $\beta$ : goto Stop.

$$
\begin{aligned}
\mathbf{w}(l+1) & =\mathbf{w}(l)+\mu \mathbf{d}(l) \\
\mathbf{w}(l+1) & =\frac{\mathbf{w}(l+1)}{\|\mathbf{w}(l+1)\|} \\
\phi_{l} & =\frac{\left\|\nabla P_{E}(\mathbf{w}(l+1))\right\|^{2}}{\left\|\nabla P_{E}(\mathbf{w}(l))\right\|^{2}} \\
\mathbf{d}(l+1) & =\phi_{l} \mathbf{d}(l)-\nabla P_{E}(\mathbf{w}(l+1))
\end{aligned}
$$

$l=l+1$, goto Loop.

Stop. $\mathbf{w}(l)$ is the solution.

At a local minimum, $\left\|\nabla P_{E}(\mathbf{w})\right\|=0$. Therefore, the small positive scalar $\beta$ determines the accuracy of the solution obtained. A gradient algorithm can, in general, find a local minimum of $P_{E}(\mathbf{w})$. To ensure a unique global solution, certain constraints can be added to the optimization problem, as shown in [7], at a possible cost that the solution found may deviate slightly from the true MBER solution.

In general, the global MBER solutions are infinitely many, and they form a half hyperplane in the $M$-dimensional weight space. The normalization (25) has an effect of fixing the solution to a unique unit-length one. Consider the following simplest example with two equal-power users and two chips per bit. The two chip codes are $(+1,+1)$ and $(+1,-1)$, respectively, and the transfer function of the CIR is $H(z)=1+0.8 z^{-1}+0.6 z^{-2}$. The SNR for user $1 \mathrm{SNR}_{1}$, is $25 \mathrm{~dB}$. The $\log _{10}$ (BER) surface for user 1 is plotted in Fig. 2. For this example, there are only global minimum solutions, and all the MBER solutions form a half line [half hyperplane in the two-dimensional (2-D) space]. The point marked in the MBER solution half line is the unit-length one. The MMSE solution for this example is also depicted in Fig. 2, where it can be seen that the MMSE solution is very different from the MBER ones. In fact, for the MMSE solution, $\log _{10}(\mathrm{BER})=-3.88$, whereas for an MBER one, $\log _{10}(\mathrm{BER})=-5.56$.

\section{AdAPTive MBER Linear Multiuser Detector}

The key to developing an effective adaptive algorithm is the pdf $p_{y}\left(y_{s} ; \mathbf{w}\right)$ of the signed decision variable. Kernel density estimation is known to produce reliable pdf estimates with short data records and, in particular,is extremely natural in dealing with Gaussian mixtures [20], [21]. Given a block of $K$ training 


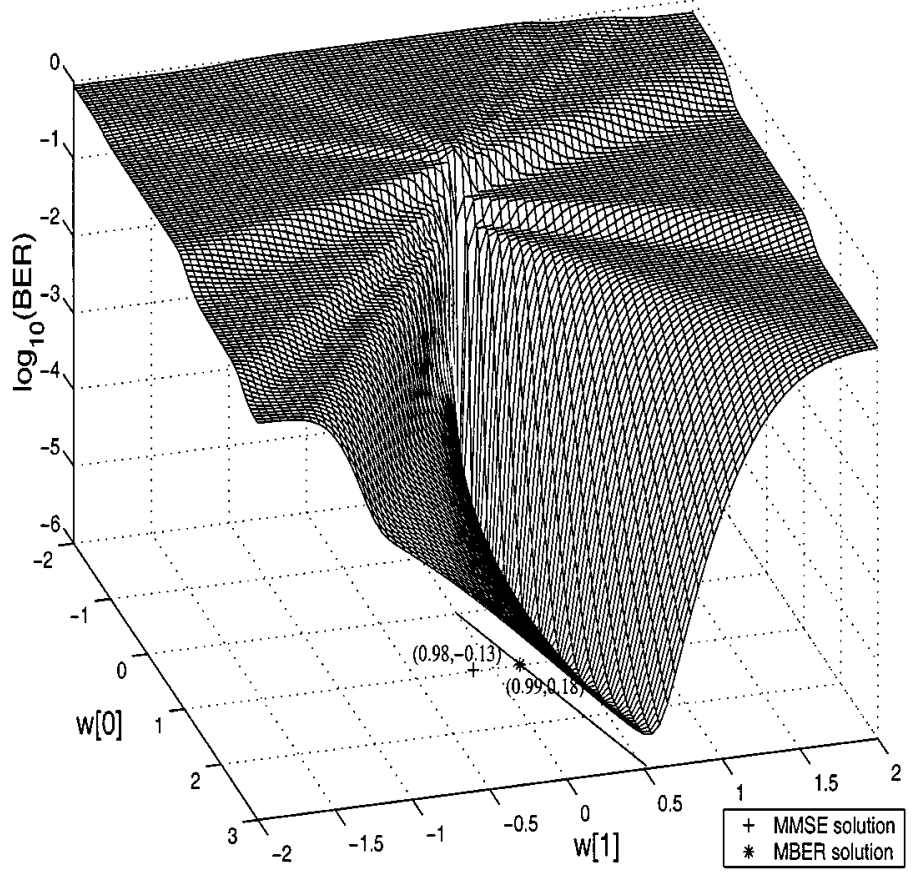

Fig. 2. Bit error rate surface of user-one detector for a simple two-user system with two chips per bit and $\mathrm{SNR}_{1}=25 \mathrm{~dB}$.

samples $\left\{\mathbf{r}(k), b_{i}(k)\right\}$, a kernel density estimate of the pdf is given by

$$
\begin{aligned}
\hat{p}_{y}\left(y_{s} ; \mathbf{w}\right)= & \frac{1}{K \sqrt{2 \pi} \rho_{n} \sqrt{\mathbf{w}^{T} \mathbf{w}}} \\
& \times \sum_{k=1}^{K} \exp \left(-\frac{\left(y_{s}-\operatorname{sgn}\left(b_{i}(k)\right) y(k ; \mathbf{w})\right)^{2}}{2 \rho_{n}^{2} \mathbf{w}^{T} \mathbf{w}}\right)
\end{aligned}
$$

where the radius parameter $\rho_{n}$ is related to the noise standard deviation $\sigma_{n}$. A lower bound of $\rho_{n}$ is [20]

$$
\rho_{n}=\left(\frac{4}{3 K}\right)^{\frac{1}{5}} \sigma_{n}
$$

From the estimated error probability

$$
\hat{P}_{E}(\mathbf{w})=\int_{-\infty}^{0} \hat{p}_{y}\left(y_{s} ; \mathbf{w}\right) d y_{s}
$$

$\nabla \hat{P}_{E}(\mathbf{w})$ can be calculated as

$$
\begin{aligned}
\nabla \hat{P}_{E}(\mathbf{w})= & \frac{1}{\sqrt{2 \pi} \rho_{n}}\left(\frac{\mathbf{w w}^{T}-\mathbf{w}^{T} \mathbf{w} \mathbf{I}}{\left(\mathbf{w}^{T} \mathbf{w}\right)^{\frac{3}{2}}}\right) \\
& \times \sum_{k=1}^{K} \exp \left(-\frac{y^{2}(k ; \mathbf{w})}{2 \rho_{n}^{2} \mathbf{w}^{T} \mathbf{w}}\right) \operatorname{sgn}\left(b_{i}(k)\right) \mathbf{r}(k) .
\end{aligned}
$$

Thus, block adaptive gradient algorithms can readily be developed by substituting $\nabla P_{E}(\mathbf{w})$ with $\nabla \hat{P}_{E}(\mathbf{w})$ in the steepest-descent or conjugate gradient updating mechanisms.

\section{A. LBER Stochastic Adaptive Algorithm}

Our aim is, however, to develop a LMS-style adaptive algorithm with sample-by-sample adjustment, as in [15] and [16].
The LMS algorithm can be viewed as replacing the ensemble average of the gradient in its related steepest-descent gradient algorithm by a single data-point estimate of the gradient. In a similar manner, at sample $k$, a point estimate of the pdf is simply

$$
\begin{aligned}
\hat{p}_{y}\left(y_{s}, k ; \mathbf{w}\right)= & \frac{1}{\sqrt{2 \pi} \rho_{n} \sqrt{\mathbf{w}^{T} \mathbf{w}}} \\
& \times \exp \left(-\frac{\left(y_{s}-\operatorname{sgn}\left(b_{i}(k)\right) y(k ; \mathbf{w})\right)^{2}}{2 \rho_{n}^{2} \mathbf{w}^{T} \mathbf{w}}\right) .
\end{aligned}
$$

Using the instantaneous or stochastic gradient

$$
\begin{aligned}
\nabla \hat{P}_{E}(k ; \mathbf{w})= & \frac{1}{\sqrt{2 \pi} \rho_{n}}\left(\frac{\mathbf{w w}^{T}-\mathbf{w}^{T} \mathbf{w} \mathbf{I}}{\left(\mathbf{w}^{T} \mathbf{w}\right)^{\frac{3}{2}}}\right) \\
& \times \exp \left(-\frac{y^{2}(k ; \mathbf{w})}{2 \rho_{n}^{2} \mathbf{w}^{T} \mathbf{w}}\right) \operatorname{sgn}\left(b_{i}(k)\right) \mathbf{r}(k)
\end{aligned}
$$

and rescaling after each update to ensure $\mathbf{w}^{T}(k) \mathbf{w}(k)=1$ gives rise to a LMS style stochastic algorithm

$$
\begin{aligned}
\mathbf{w}(k+1)= & \mathbf{w}(k)+\frac{\mu}{\sqrt{2 \pi} \rho_{n}} \exp \left(-\frac{y^{2}(k ; \mathbf{w}(k))}{2 \rho_{n}^{2}}\right) \\
& \times \operatorname{sgn}\left(b_{i}(k)\right)(\mathrm{r}(k)-\mathbf{w}(k) y(k ; \mathbf{w}(k)))
\end{aligned}
$$

which we refer to as the LBER algorithm.

Two important issues for any stochastic gradient adaptive MBER algorithm are the convergence speed and steady-state BER misadjustment (with respect to the optimal MBER). A theoretical analysis of these two properties for the LBER (33) is extremely complex and is still under investigation. We will use computer simulation to study these two properties.

\section{B. Comparison with the DMBER and AMBER Adaptive Algorithms}

The motivation of the above LBER multiuser detector is different from those of the existing DMBER and AMBER detectors [8], [9]. For the purpose of a comparison, we present a modified version of the DMBER adaptive algorithm reported in [8]. Define the one-sample decision distortion measure ${ }^{3}$

$$
\zeta(k ; \mathbf{w})=\frac{1}{2}\left|b_{i}(k)-\hat{b}_{i}(k)\right|
$$

Notice that this distortion measure is a function of $\mathbf{w}$, as $\hat{b}_{i}(k)=\operatorname{sgn}\left(\mathbf{w}^{T} \mathbf{r}(k)\right)$. Obviously, $\zeta(k ; \mathbf{w})=\hat{P}_{E}(k ; \mathbf{w})$ is the one-sample error probability. The following difference approximation for the gradient of $\zeta(k ; \mathbf{w})$ is adopted as

$$
\mathbf{x}(k ; \mathbf{w})=\left[x_{1}(k ; \mathbf{w}) \ldots x_{M}(k ; \mathbf{w})\right]^{T}
$$

with

$$
\begin{array}{r}
x_{j}(k ; \mathbf{w})=\frac{1}{2 c_{k}}\left(\zeta\left(k ; \mathbf{w}+c_{k} \mathbf{e}_{j}\right)-\zeta\left(k ; \mathbf{w}-c_{k} \mathbf{e}_{j}\right)\right) \\
1 \leq j \leq M
\end{array}
$$

${ }^{3}$ In [8], the distortion measure is defined in terms of two samples: one for $b_{i}(k)=-1$ and the other for $b_{i}(k+1)=1$, which lead to some complications in sample-by-sample adaptation. Our modification has a nature and simple adaptive implementation and is equivalent to the original formulation. 
where $c_{k}=c_{0} k^{-1 / 4}$ for some $c_{0}>0$, and $\mathbf{e}_{j}$ denotes the $j$ th coordinate unit vector. The DMBER adaptive algorithm takes the form

$$
\mathbf{w}(k+1)=\mathbf{w}(k)-\alpha \mathbf{x}(k ; \mathbf{w}(k))
$$

where $\alpha$ is an adaptive step size.

In theory, the DMBER should work, as it attempts to minimize the BER directly. As a difference approximation of the stochastic gradient is used, the algorithm does not rely on the assumption of a Gaussian channel noise at a cost of increased complexity. (The DMBER has a complexity of $O\left(M^{2}\right)$, whereas the AMBER and LBER has a complexity of $O(M)$.) However, in practice, its rate of convergence is very slow after the weight vector has reached the region of small error rate. This is obvious since $\mathrm{x}(k ; \mathrm{w})$ will be zero most of the time.

A more efficient stochastic gradient adaptive algorithm is the AMBER [9], which can be expressed as

$$
\mathbf{w}(k+1)=\mathbf{w}(k)+\mu I(k ; \mathbf{w}(k)) \operatorname{sgn}(e(k ; \mathbf{w}(k))) \mathbf{r}(k)
$$

with the error signal

$$
e(k ; \mathbf{w})=b_{i}(k)-y(k ; \mathbf{w})
$$

and the indicator function

$$
I(k ; \mathbf{w})=\frac{1}{2}\left(1-\operatorname{sgn}\left(b_{i}(k) y(k ; \mathbf{w})-\tau\right)\right)
$$

where $\tau$ is a non-negative threshold parameter. In terms of the algorithm-tuning requirements, the two adaptive algorithms [the LBER (33) and AMBER (38)] are similar. The former requires the tuning of the adaptive gain $\mu$ and kernel width $\rho_{n}$, whereas the latter needs the tuning of the adaptive gain $\mu$ and threshold parameter $\tau$.

The following comparison of the two adaptive mechanisms (the LBER and AMBER) can be made [15], [16]. The algorithm (38) in its simplest form has $\tau=0$, and it only updates when a decision error is observed. When the algorithm is initialized, it is unlikely to separate all the noise-free states $\mathbf{r}_{j}$ correctly. Thus, the indicator function $I(k ; \mathbf{w})$ will be on most of the time, in which case, it is equivalent to the signed-error LMS algorithm [10]. When the algorithm has converged to a point where it can separate the noise-free states correctly, the probability of the algorithm updating may be low because in this region, errors will be predominated by noise, and hence, further convergence may be slow. Introducing the threshold $\tau$ essentially defines a region around decision boundary where the algorithm will continue to update, even when errors do not occur. This region is defined by

$$
\left|\mathbf{w}^{T} \mathbf{r}(k)\right|<\tau .
$$

In the algorithm (33), the effect of the distance from the decision boundary is controlled by the exponential term $\exp \left(-y^{2}(k ; \mathbf{w}) /\left(2 \rho_{n}^{2}\right)\right)$. This can be viewed as a soft distance measure. The size of an update is a continuous and decreasing function of the distance from the boundary. The distance is scaled by the kernel width $\rho_{n}$, which in turn is a function of the noise root mean square $\sigma_{n}$.

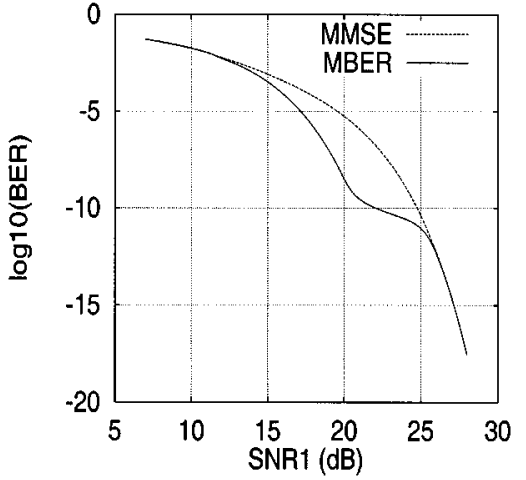

Fig. 3. Linear detector BERs for user 1 of Example 1. $\mathrm{SNR}_{1}=\mathrm{SNR}_{2}$.

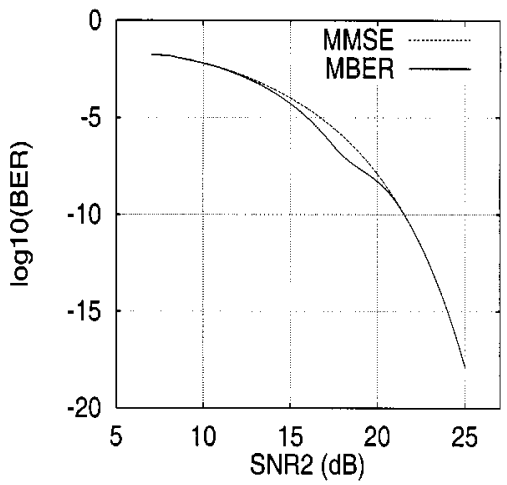

Fig. 4. Linear detector BERs for user 2 of Example $1 . \mathrm{SNR}_{1}=\mathrm{SNR}_{2}$.

\section{Simulation Results}

Computer simulation was conducted to investigate the convergence speed and steady-state BER misadjustment for the three stochastic adaptive algorithms. For the DMBER algorithm, a fixed step-size $\alpha$ was used with a time-varying difference step $c_{k}=c_{0} k^{-1 / 4}$ (as was used in [8]). For the AMBER algorithm, the threshold $\tau$ was fixed with a time-varying adaptive step-size $\mu$ given by

$$
\mu_{k}=\mu_{0} k^{-1} \text {. }
$$

The LBER algorithm had a constant width $\rho_{n}$ and employed the time-varying adaptive step size, as given by (42). The two algorithm parameters $c_{0}$ and $\alpha$ for the DMBER, $\mu_{0}$ and $\tau$ for the AMBER, and $\mu_{0}$ and $\rho_{n}$ for the LBER, were chosen to give an adequate combined result of convergence rate and steady-state error for the respective algorithm.

Example 1: A two-user system with four chips per symbol was used in the simulation. The code sequences of the two users were $(+1,+1,-1,-1)$ and $(+1,-1,-1,+1)$, respectively, and the transfer function of the CIR was

$$
H(z)=1.0+0.25 z^{-1}+0.5 z^{-3} .
$$

The two users had equal signal power, that is, $\mathrm{SNR}_{1}$ was equal to $\mathrm{SNR}_{2}$. Figs. 3 and 4 depict the linear detector BERs for the two users, respectively. The BER formula (20) was used with the detector weight vector set to the MMSE and MBER solutions, respectively, to produce the corresponding error rate curves. For this example, the difference between the MMSE and MBER 


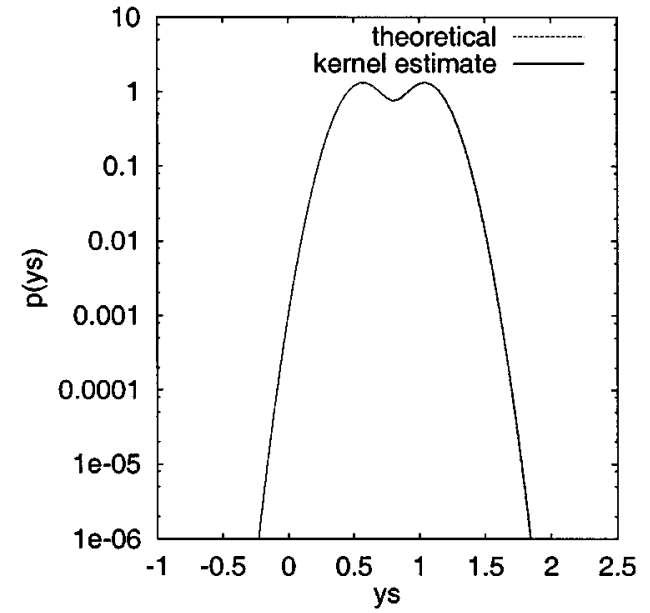

Fig. 5. Distribution of the signed decision variable for user 1 of Example 1. $\mathrm{SNR}_{1}=\mathrm{SNR}_{2}=16.5 \mathrm{~dB}$, the detector weight vector was set to the MBER solution, and the kernel estimate was constructed from 100 data samples.

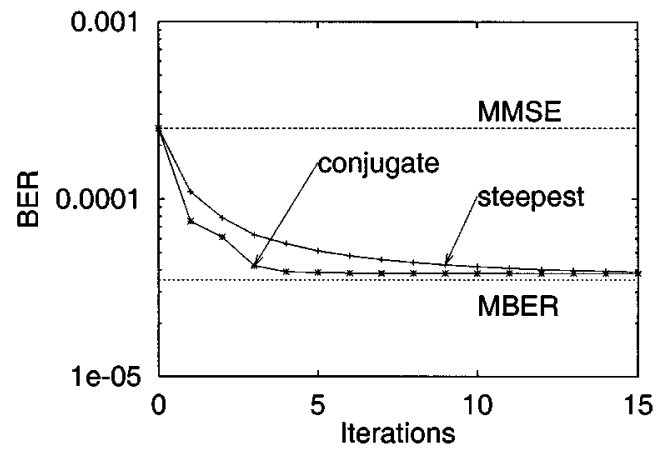

Fig. 6. Convergence behaviors of the two block adaptive MBER algorithms for user 1 of Example 1. Length of the data block is 100 , and $\mathrm{SNR}_{1}=\mathrm{SNR}_{2}=$ $16.5 \mathrm{~dB}$.

solutions for user 1 is significant for the range of $\mathrm{SNR}_{1}=14$ to $26 \mathrm{~dB}$.

The kernel density estimate (27) constructed from 100 data samples at $\mathrm{SNR}_{1}=\mathrm{SNR}_{2}=16.5 \mathrm{~dB}$ is compared with the true pdf (19) in Fig. 5 for user 1, where the detector weight vector was set to the MBER solution. For this example, the kernel estimate and the true density are indistinguishable. Using the constructed kernel density estimate with the detector weight vector $\mathbf{w}$, the block adaptive steepest-descent and conjugate gradient algorithms were applied to find the MBER solutions, and the two iterative procedures are illustrated in Fig. 6. It can be seen that starting from the MMSE solution, the block adaptive conjugate gradient algorithm took fouriterations to converge to the MBER solution.

The three stochastic gradient adaptive algorithms (the DMBER, AMBER and LBER) were applied to user 1 with $\mathrm{SNR}_{1}=\mathrm{SNR}_{2}=19 \mathrm{~dB}$ and the initial weight vector set to the MMSE solution. For the DMBER, $c_{0}=2.0$ and $\alpha=0.01$ was used. The AMBER used $\mu_{0}=0.5$ and $\tau=0.8$, whereas the LBER had $\mu_{0}=1.0$ and $\rho_{n}^{2}=0.11(\approx 9 \times$ noise variance $)$. These algorithm parameters were found in simulation to be adequate for the respective algorithm. The convergence performance of these three algorithms are shown in Fig. 7, where the results were averaged on 100 runs. The standard LMS with

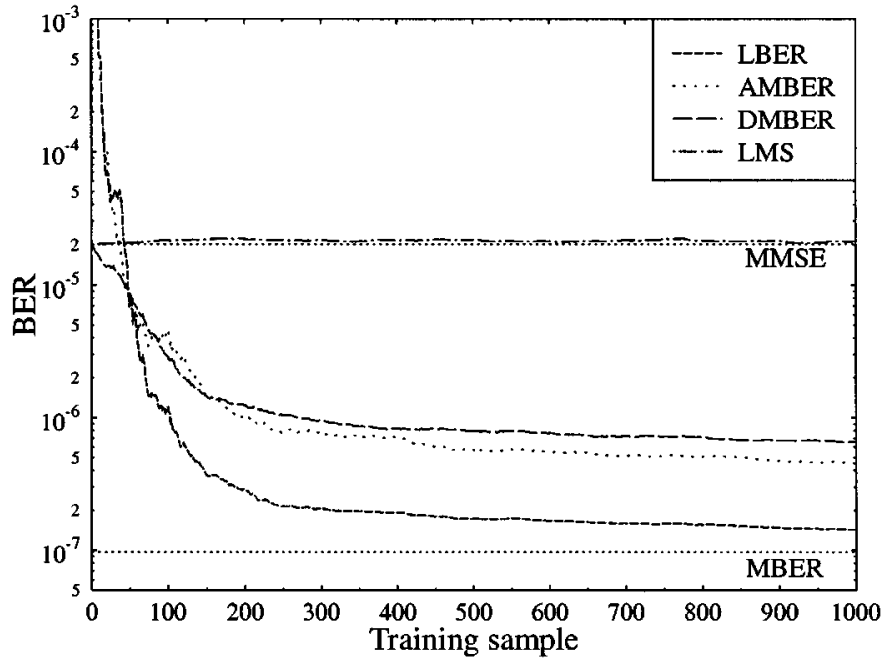

Fig. 7. Learning curves of the three stochastic adaptive MBER algorithms and the LMS algorithm for user 1 of Example $1 . \mathrm{SNR}_{1}=\mathrm{SNR}_{2}=19 \mathrm{~dB}$.

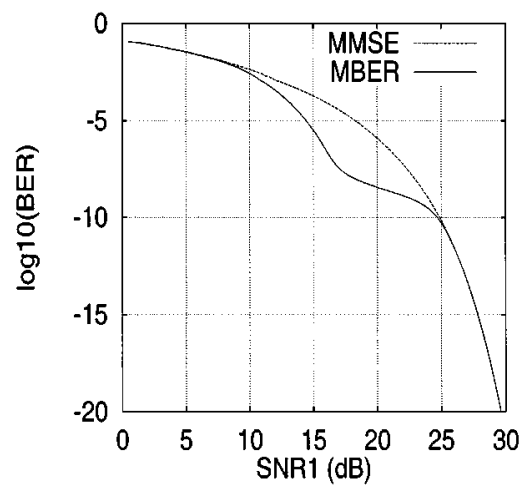

Fig. 8. Linear detector BERs for user 1 of Example 2. $\mathrm{SNR}_{i}, 1 \leq i \leq 4$ are identical.

an adaptive gain 0.01 is also depicted in Fig. 7. The value of the learning curve at $k$ is the true BER $P_{E}(\mathbf{w}(k))$ of (20) for the given weight vector $\mathbf{w}(k)$ and not any approximation. For this example, it can be seen that the proposed LBER algorithm is superior over the other two adaptive MBER algorithms in terms of convergence speed and steady-state error.

Example 2: This was a four-user system with 8 chips per symbol. The code sequences for the four users were $(+1,+1,+1,+1,-1,-1,-1,-1), \quad(+1,-1$, $+1,-1,-1,+1,-1,+1),(+1,+1,-1,-1,-1,-1,+1,+1)$ and $(+1,-1,-1,+1,-1,+1,+1,-1)$, respectively, and the transfer function of the CIR was

$$
H(z)=0.4+0.7 z^{-1}+0.4 z^{-2} .
$$

The four users had equal signal power. Figs. 8-10 depict the linear detector BERs for the four users, respectively. For users 2 and 4, the MMSE and MBER solutions are indistinguishable.

Fig. 11 shows the kernel estimate and true pdf of the signed decision variable for user 1 at $\mathrm{SNR}_{i}=16 \mathrm{~dB}(1 \leq i \leq 4)$. The pdf estimate was constructed from 1500 data samples, and the detector weight vector was set to the MBER solution. It can be seen that for this example, the kernel estimate approximates the true density reasonably well. Based on the kernel 


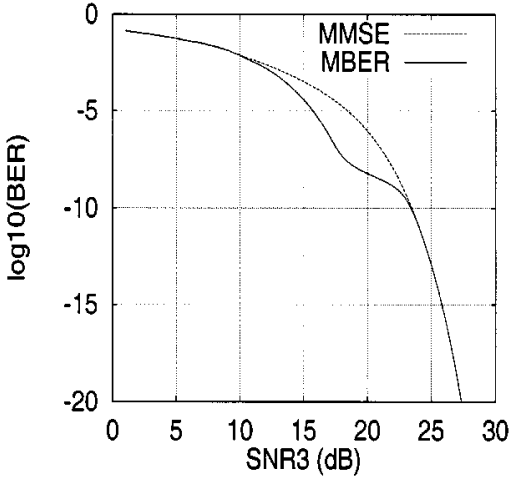

Fig. 9. Linear detector BERs for user 3 of Example 2. $\mathrm{SNR}_{i}, 1 \leq i \leq 4$ are identical.

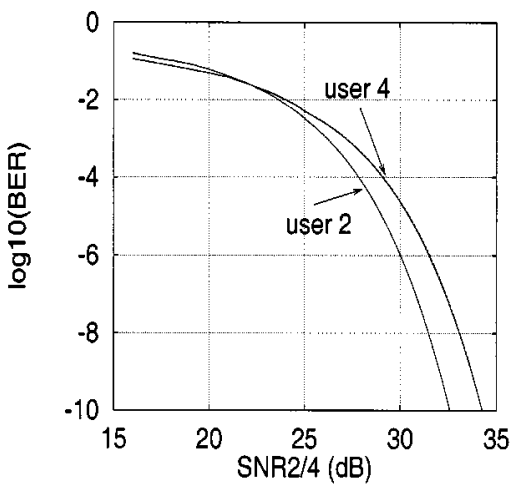

Fig. 10. Linear detector BERs for users 2 and 4 of Example 2. $\mathrm{SNR}_{i}, 1 \leq$ $i \leq 4$ are identical. The MMSE and MBER solutions for these two users are indistinguishable.

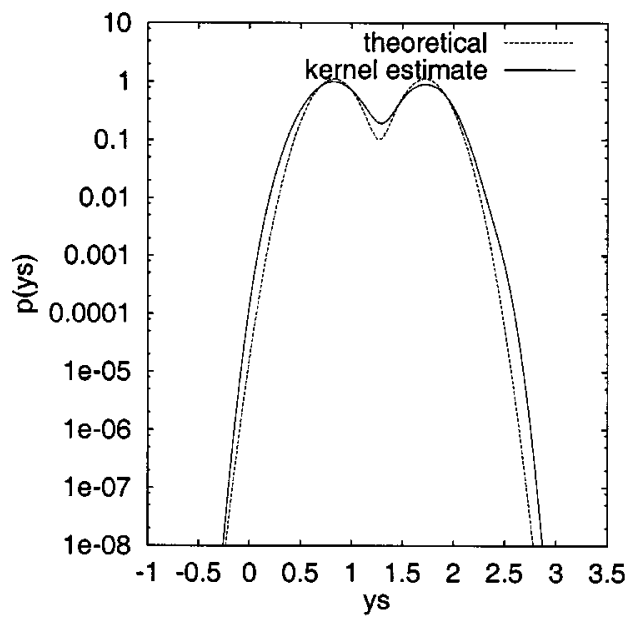

Fig. 11. Distribution of the signed decision variable for user 1 of Example 2. $\mathrm{SNR}_{i}=16 \mathrm{~dB}$ for $1 \leq i \leq 4$, the detector weight vector was set to the MBER solution, and the kernel estimate was constructed from 1500 data samples.

density estimate, the iterative procedures of the block adaptive steepest-descent and conjugate gradient algorithms are depicted in Fig. 12. It can be seen that starting from the MMSE solution, the block adaptive conjugate gradient algorithm took five iterations to converge to a near-MBER solution.

The three stochastic gradient adaptive algorithms were tested for user 1 at $\mathrm{SNR}_{i}=15 \mathrm{~dB}$, and $1 \leq i \leq 4$ with the initial weight vector set to the MMSE solution. The two algorithm

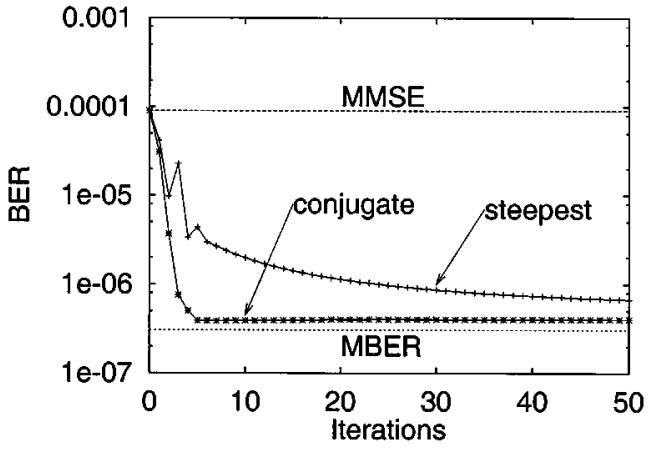

Fig. 12. Convergence behaviors of the two block adaptive MBER algorithms for user 1 of Example 2. Length of the data block is 1500, and $\mathrm{SNR}_{i}=16 \mathrm{~dB}$ for $1 \leq i \leq 4$.

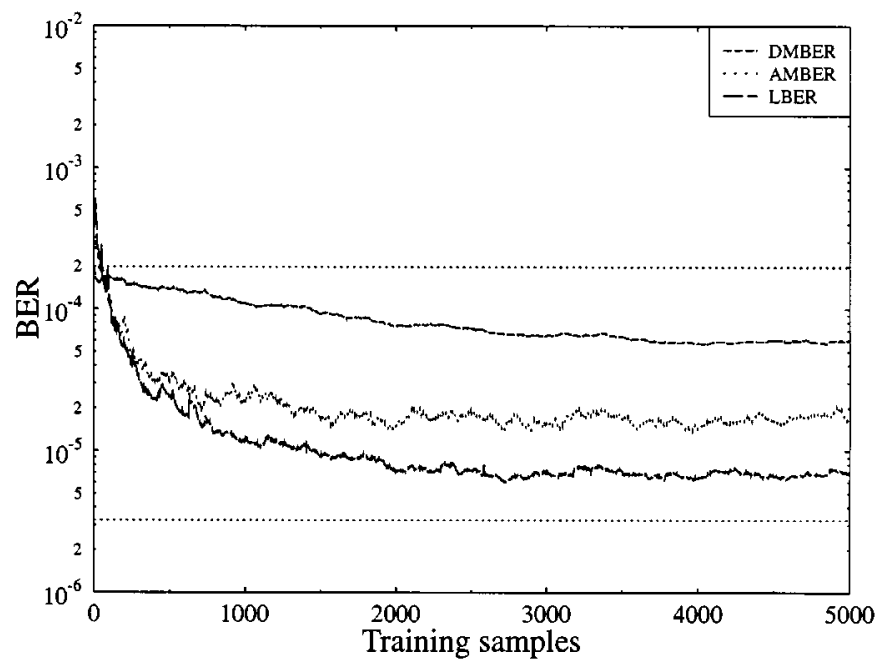

Fig. 13. Learning curves of the three stochastic adaptive MBER algorithms for user 1 of Example $2 . \mathrm{SNR}_{i}=15 \mathrm{~dB}$ for $1 \leq i \leq 4$.

parameters were found to be $c_{0}=2.0$ and $\alpha=0.01$ for the DMBER, and $\mu_{0}=0.05$ and $\tau=0.8$ for the AMBER. For this example, the LBER used $c_{k}=0.2 k^{-1 / 4}$ and $\rho_{n}^{2}=0.06(\approx 2 \times$ noise variance). The learning curves of these three algorithms are given in Fig. 13, where the results were averaged on 50 runs. For this example, the very slow convergence of the DMBER is apparent when the BER is below $10^{-4}$. It can also be seen that the LBER algorithm (33) has a superior performance over the AMBER algorithm (38).

\section{CONCLUSION}

The MBER linear multiuser detection has been considered for DS-CDMA systems. Motivated from the kernel density estimation of the BER as a smooth function of the training data, block-based adaptive gradient algorithms have been developed to realize the MBER linear multiuser detector. This has further led to the derivation of a LMS-style adaptive MBER algorithm for linear multiuser detectors. A desired feature of this stochastic gradient algorithm is that the amount of the weight updating is a continuous and decreasing function of the distance from the decision boundary. Simulation results indicate that this adaptive MBER detector outperforms an existing LMS-style MBER algorithm called the AMBER in terms of convergence speed and 
steady-state BER misadjustment. Theoretical analysis of convergence and steady-state misadjustment for the proposed new adaptive MBER algorithm is still under investigation.

\section{REFERENCES}

[1] Z. Xie, R. T. Short, and C. K. Rushforth, "A family of suboptimum detectors for coherent multiuser communications," IEEE J. Select. Area Commun., vol. 8, pp. 683-690, Apr. 1990.

[2] U. Madhow and M. L. Honig, "MMSE interference suppression for direct-sequence spread-spectrum CDMA," IEEE Trans. Commun., vol. 42, pp. 3178-3188, Dec. 1994.

[3] S. L. Miller, "An adaptive direct-sequence code-division multiple-access receiver for multiuser interference rejection," IEEE Trans. Commun. vol. 43, pp. 1746-1755, Feb./Mar./Apr. 1995.

[4] H. V. Poor and S. Verdú, "Probability of error in MMSE multiuser detection," IEEE Trans. Inform. Theory, vol. 43, pp. 858-871, Mar. 1997.

[5] G. Woodward and B. S. Vucetic, "Adaptive detection for DS-CDMA," Proc. IEEE, vol. 86, pp. 1413-1434, July 1998

[6] N. B. Mandayam and B. Aazhang, "Gradient estimation for sensitivity analysis and adaptive multiuser interference rejection in code-division multi-access systems," IEEE Trans. Commun., vol. 45, pp. 848-858, July 1997

[7] X. F. Wang, W. S. Lu, and A. Antoniou, "Constrained minimum-BER multiuser detection," in Proc. ICASSP, vol. 5, Phoenix, AZ, May 14-18, 1999, pp. 2603-2606.

[8] I. N. Psaromiligkos, S. N. Batalama, and D. A. Pados, "On adaptive minimum probability of error linear filter receivers for DS-CDMA channels," IEEE Trans. Commun., vol. 47, pp. 1092-1102, July 1999.

[9] C. C. Yeh, R. R. Lopes, and J. R. Barry, "Approximate minimum biterror rate multiuser detection," in Proc. Globecom, Sydney, Australia, Nov. 1998 , pp. 3590-3595

[10] R. Sharma, W. A. Sethares, and J. A. Bucklew, "Asymptotic analysis of stochastic gradient-based adaptive filtering algorithms with general cost functions," IEEE Trans. Signal Processing, vol. 44, pp. 2186-2194, Sept. 1996.

[11] E. Shamash and K. Yao, "On the structure and performance of a linear decision feedback equalizer based on the minimum error probability criterion," in Proc. ICC, 1974, pp. 25F1-25F5.

[12] S. Chen, E. S. Chng, B. Mulgrew, and G. Gibson, "Minimum-BER linear-combiner DFE," in Proc. ICC, vol. 2, Dallas, TX, 1996, pp. 1173-1177.

[13] S. Chen, B. Mulgrew, E. S. Chng, and G. Gibson, "Space translation properties and the minimum-BER linear-combiner DFE," Proc. Inst Elect. Eng.-Commun., vol. 145, no. 5, pp. 316-322, 1998.

[14] S. Chen and B. Mulgrew, "The minimum-SER linear-combiner decision feedback equalizer," Proc. Inst. Elect. Eng.-Commun., vol. 146, no. 6, pp. 347-353, 1999 .

[15] B. Mulgrew and S. Chen, "Adaptive minimum-BER decision feedback equalisers for binary signalling," Signal Process., 1999, submitted for publication.

[16] - "Stochastic gradient minimum-BER decision feedback equalisers," in Proc. IEEE Symp. Adaptive Syst. Signal Process., Commun., Contr., Lake Louise, AB, Canada, Oct. 1-4, 2000, pp. 93-98.

[17] C. C. Yeh and J. R. Barry, "Approximate minimum bit-error rate equalization for binary signaling," in Proc. ICC, vol. 2, Montreal, PQ, Canada, 1997, pp. 1095-1099.

[18] B. Mulgrew, "Nonlinear signal processing for adaptive equalization and multi-user detection," in Proc. EUSIPCO, Rhodes, Greece, Sept. 1998, pp. 537-544.

[19] M. S. Bazaraa, H. D. Sherali, and C. M. Shetty, Nonlinear Programming: Theory and Algorithms. New York: Wiley, 1993.

[20] B. W. Silverman, Density Estimation. London, U.K.: Chapman \& Hall, 1996.

[21] A. W. Bowman and A. Azzalini, Applied Smoothing Techniques for Dato Analysis. Oxford, U.K.: Oxford Univ. Press, 1997.

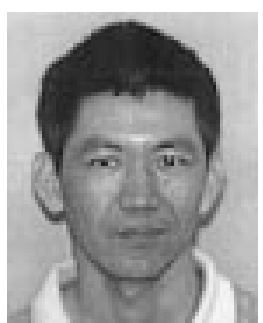

Sheng Chen (SM'97) received the Ph.D. degree in control engineering from the City University, London, U.K., in 1986.

From 1986 to 1999 , he held various research and academic appointments at the Universities of Sheffield, Edinburgh, and Portsmouth, U.K. Since 1999, he has been with the Department of Electronics and Computer Science, the University of Southampton, U.K., where he currently holds a post of Readership in Communications Signal Processing. His research interests include modeling and identification of nonlinear systems, adaptive nonlinear signal processing, artificial neural network research, finite-precision digital controller design, evolutionary computation methods, and optimization. He has published over 140 research papers.

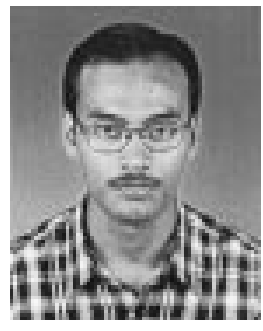

Ahmad K. Samingan received the B.Eng. (Hons.) degree in electronic engineering from the University of Southampton, U.K., in 1999. He is currently with the Communication Group, Department of Electronic and Computer Science, the University of Southampton, where he is pursuing the Ph.D. degree.

His research interests cover the field of CDMA multiuser detection, particularly in the areas of adaptive multiuser detection and support vector machine (SVM) based multiuser detection.

Mr. Samingan was named a Zepler Prize winner in 1998 for his excellent academic achievement.

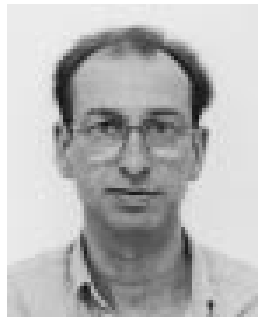

Bernard Mulgrew (M'89) received the B.Sc. degree in electrical and electronic engineering in 1979 from Queen's University, Belfast, U.K. He received the Ph.D. degree in 1987 for the University of Edinburgh, U.K.

After graduation, he worked for four years as a Development Engineer with the Radar Systems Department, GEC-Marconi Avionics, Edinburgh, U.K. From 1983 to 1986, he was a research associate with the Department of Electrical Engineering, University of Edinburgh. He became a lecturer in 1986 and was promoted to senior lectureship and readership in 1994 and 1996, respectively. He was elected to the Personal Chair in Signals and Systems in 1999. His research interests are in adaptive signal processing and estimation theory and in their application to radar, audio, and communications systems. He is a co-author of three books on signal processing, and has published over 150 research papers.

Dr. Mulgrew is a member of EURASIP, the IEE, and the Audio Engineering Society.

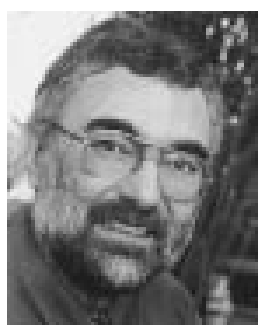

Lajos Hanzo has held various research and academic posts in Hungary, Germany, and the U.K Since 1986, he has been with the Department of Electronics and Computer Science, University of Southampton, U.K., where he currently holds the chair in telecommunications. He co-authored five books on mobile radio communications, published over 200 research papers, organized and chaired conference sessions, presented overview lectures, and was awarded a number of distinctions. Currently, he is managing a research team, working on a range of research projects in the field of wireless multimedia communications under the auspices of the Engineering and Physical Sciences Research Council (EPSRC) U.K., the European IST Programme, and the Mobile Virtual Centre of Excellence (VCE). 